\title{
Glottopol
}

Revue de sociolinguistique en ligne

$35 \mid 2021$

La langue à l'école, de l'institution à la classe : quelles conceptions, quelles normes, pour quels usages?

\section{Ursula Reutner, Manuel des francophonies}

Berlin/Boston, de Gruyter, 745 p.

\section{Katrin Pfadenhauer}

\section{CpenEdition}

Journals

Édition électronique

URL : https://journals.openedition.org/glottopol/308

DOI : $10.4000 /$ glottopol.308

ISSN : $1769-7425$

Éditeur

Presses universitaires de Rouen et du Havre

Référence électronique

Katrin Pfadenhauer, «Ursula Reutner, Manuel des francophonies», Glottopol [En ligne], 35 | 2021, mis en ligne le 01 janvier 2021, consulté le 02 octobre 2021. URL : http://journals.openedition.org/glottopol/ 308 ; DOI : https://doi.org/10.4000/glottopol.308 


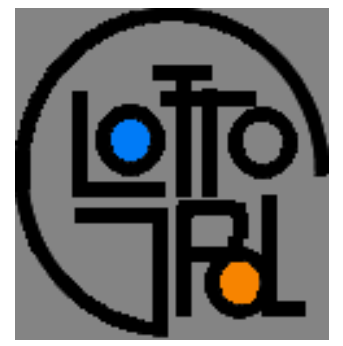

\section{GLOTTOPOL}

Revue de sociolinguistique en ligne $\mathrm{n}^{\circ} 35$ - janvier 2021

\section{La langue à l'école, de l'institution à la classe : quelles conceptions, quelles normes, pour quels usages?}

\section{SOMMAIRE}

Catherine Delarue-Breton et Élisabeth Bautier : Introduction

Catherine Delarue-Breton : Des conceptions scolaires de la langue en tension : l'exemple du programme de 2015 pour l'école primaire et le collège

Véronique Miguel-Addisu : "Sport de riche je l'ai changé en sport élitiste » : plurilinguisme et variation dans la langue de l'école du point de vue des élèves

Claire Colombel-Teuira, Véronique Fillol : Variation et approche polynomique : pour une conception plurielle de la langue à l'école calédonienne

Daphné Bloch : Pratiques langagières, situations pédagogiques et construction d'inégalités d'apprentissage à Madagascar

Samatar Abdallah Doualeh : Le français langue de l'école djiboutienne ou l'hégémonie de la norme exogène

Élise Vinel, Élisabeth Bautier : Des discours des élèves sur l'orthographe aux pratiques des enseignants, analyse d'entretiens métagraphiques

Thierry Pagnier, Belinda Lavieu-Gwozdz: Regards sur le discours scolaire: saisir des conceptions de la langue et de son enseignement

Jacques Crinon, Georges Ferone, Hélène Font : Les enseignants et l'orthographe, une enquête au cycle 3

Christel Troncy : Les enseignants, la norme scolaire et la pluralité langagière dans deux écoles immersives à programme français en Californie. Dynamiques des attitudes et des pratiques lors d'une recherche collaborative

Erwan Le Pipec: L'école, planche de salut du breton?

Argia Olçomendy : La langue basque dans les instructions officielles (1976-2019)

\section{Compte rendu de lecture}

Par Katrin Pfadenhauer: Ursula Reutner (Ed.), 2017, Manuel des francophonies, Berlin/Boston, de Gruyter, 745 p.

Par Robert Fournier: Shana Poplack, 2018, Borrowing: Loanwords in the speech community and in the grammar. New York: Oxford University Press ; xxi, $246 \mathrm{p}$.

Par Marie-Jeanne Verny: Catherine Adam, 2020, Bilinguisme scolaire. Familles, écoles, identités en Bretagne, éd. Peter Lang, coll. «Langue, multilinguisme et changement social », Berlin. 


\section{COMPTE RENDU}

Ursula Reutner (Ed.), 2017, Manuel des francophonies, Berlin/Boston, de Gruyter, 745 p.

par Katrin Pfadenhauer

Université de Bayreuth (Allemagne)

Éditer un autre manuel sur la francophonie représente sans doute un défi. Rien que le titre de l'ouvrage laisse supposer que l'approche choisie par l'éditrice allemande Ursula Reutner entend en effet se distinguer des œuvres déjà existantes sur le même sujet. Celui-ci n'évoque pas une francophonie, mais des francophonies - usage insolite du terme, comme l'admet Reutner dans l'introduction (p. 1) - mettant ainsi en avant la pluralité linguistique au sein de l'espace francophone. Un simple regard à la table de matières de ce livre de référence d'environ 750 pages suffit pour se rendre compte que l'objectif central de l'ouvrage est de proposer une description de l'espace francophone dans sa totalité dont la France ne représente, au fond, qu'une partie. Dans cette perspective, et à la différence d'autres livres publiés sur le sujet il y a une trentaine, voire une quarantaine d'années (voir, entre autres, Valdman 1979, de Robillard/Beniamino 1993, 1996), la situation linguistique en France est abordée dans la partie portant sur le français en Europe, à côté des articles traitant les pays européens limitrophes où le français fait partie du paysage linguistique (la Belgique (M. Francard), la Suisse (A. Thibault), le Luxembourg (J. Kramer/A. Willems) et la Vallée d'Aoste (R. Bauer) ${ }^{1}$ ). Par ailleurs, avec des chapitres s'intéressant à l'espace occitan et francoprovençal (C. PolzinHaumann), la Catalogne et le Corse (R. Kailuweit), l'Alsace (U. Reutner), la Bretagne (F. Broudic) et le Pays basque (J.-B. Coyos), le champ linguistique dans l'Hexagone est appréhendé dans toute sa complexité, en tenant compte de diverses situations de contact entre le français et les langues régionales.

L'organisation thématique du manuel suit des critères géographiques. Seule la dernière partie fait figure d'exception en réunissant des chapitres consacrés aux français parlés dans les iles et dans la France d'outre-mer. Elle regroupe ainsi les espaces créolophones qui se trouvent, d'un point de vue géographique, éloignés l'un de l'autre (Haïti, Guadeloupe, Martinique et Guyane dans l'Océan Atlantique et la Réunion, Maurice et Seychelles dans l'Océan Indien). Madagascar, les Comores et Mayotte (V. Randriamarotsimba), de même que la Polynésie et la Mélanésie (S. Ehrhart), sont également intégrées, en tant qu'espaces francophones de l'Océan Indien et Pacifique, dans cette partie pour des raisons liées à leur passé colonial. La structure du livre est claire et pertinente. La première partie se focalise sur la situation du français en

\footnotetext{
${ }^{1}$ La Vallée d'Aoste figure dans le sous-groupe «Pays limitrophes » du chapitre «Le français en Europe » du manuel bien que - au sens strict - il s'agit d'une région à statut spécial d'Italie et pas d'un pays dans le sens propre.
} 
Europe. Elle est suivie par des sections consacrées au français en Amérique, en Afrique et en Asie. Les francophonies ${ }^{2}$ européennes (voir ci-dessus) et américaines sont presque traitées dans leur totalité. Le lecteur trouve des contributions sur le Québec (L. Mercier/ W. Remysen/ H. Cajolet-Laganière), l'Acadie des Maritimes (A. Boudreau/ K. Gauvin), l'Ontario (J. Tennant), l'Ouest du Canada (L. Rodriguez), la Nouvelle-Angleterre (C. Fox), la Louisiane (T. A. Klingler), Haïti (D. Fattier), la Guadeloupe et la Martinique (J.-D. Bellonie/ E. Pustka) et la Guyane (S. Alby). Il en va autrement pour les pays francophones d'Afrique qui ne sont que partiellement couverts : l'Algérie (Y. Derradji) et le Maroc (F. Jablonka) pour le cas du Maghreb, la Côte d'Ivoire et le Burkina Faso (O. Boukari), le Cameroun (M. Drescher), le Burundi (C. Frey), le Sénégal (M. Daff), ainsi que les iles de Madagascar, des Comores, de Mayotte, de la Réunion, de Maurice et des Seychelles (S. Kriegel), en ce qui concerne l'Afrique subsaharienne. Le français sur le continent asiatique est pris en considération avec des chapitres sur le Liban (C. Serhan/ C. Eid/ M. Francard), le Vietnam, le Cambodge et le Laos (V. Noll). Chaque chapitre du manuel - avec l'exception de l'introduction de l'éditrice (pp. 1-6) et des premières contributions d'Ursula Reutner (Vers une typologie pluridimensionnelle des francophonies, pp. 9-64) et de Bernhard Pöll (Normes endogènes, variétés de prestige et pluralité normative, pp. 65-86) traitant des aspects généraux du sujet - s'attache à la description d'une situation linguistique particulière en suivant toujours la même structure : (1) situation démolinguistique, (2) survol historique, (3) aménagement externe (législation linguistique, les langues dans l'administration, dans l'enseignement et dans les médias), (4) spécificités du français (prononciation, morphosyntaxe et lexique) et (5) aménagement interne. Cette organisation uniforme se révèle d'une grande utilité. D'une part, elle permet la comparaison directe des critères établis dans chaque chapitre. D'autre part, en mettant en lumière le déséquilibre existant dans la description des variétés, elle sert à reprendre la discussion sur les concepts linguistiques comme norme (endogène et exogène), standard et variété (entre autres). Notamment la section (4) sur les particularités linguistiques et la section (5) sur l'aménagement interne du français dans le pays francophone en question s'avèrent être des indicateurs efficaces pour déterminer le statut du français parlé autour du globe. De plus, la section (4) révèle les problèmes et difficultés dans la description des particularités linguistiques, comme en témoigne la réflexion de l'éditrice du manuel sur la question de la définition d'une 'particularité' (p. 36) :

Revenons maintenant à l'idée de choisir le français standard de la France comme corpus d'exclusion pour définir les particularités des francophonies. Cette idée parfois intuitivement appliquée ne manque pas d'attrait pour certains, mais elle génère le problème que l'on trouve des formes qui n'appartiennent pas au standard hexagonal. Il est donc indispensable de tenir compte de celui-ci dans toute sa complexité. Mais suffit-il de classifier comme particularité francophone chaque trait linguistique courant dans le pays en question et absent dans le (reste du) français hexagonal?

À titre d'exemple, si nous regardons de plus près les français parlés dans les pays francophones du continent africain, il devient clair que la description des particularités ne peut être réalisée qu'avec beaucoup de précaution, comme l'illustrent les contributions sur la Côte d'Ivoire et le Burkina Faso, le Cameroun, le Burundi et le Sénégal. Les raisons en sont multiples. Tout d'abord, il faut souligner que, dans le cas des français parlés en Afrique, il ne s'agit pas de variétés nationales codifiées (comme c'est le cas par exemple pour le français québécois, la majorité des variétés de l'espagnol parlées sur le continent américain et le portugais du Brésil). De plus, les descriptions - sans être normatives - se concentrent surtout

\footnotetext{
${ }^{2}$ Le pluriel de francophonie est utilisé comme proposé par l'éditrice pour ainsi souligner la pluralité linguistique dans l'espace francophone.
} 
sur des aspects de prononciation et des particularités lexicales, c'est-à-dire les niveaux linguistiques les plus visibles en surface. En ce qui concerne la morphosyntaxe et les traits pragmatico-discursifs, les contributions du manuel rendent ainsi visible une lacune importante dans la recherche qui reste à être comblée. Un troisième problème est le fait que nous ne disposons pas de corpus cohérents pour les français parlés en Afrique qui puissent servir de fondement solide pour généraliser les phénomènes en question. Les matériaux disponibles (transcriptions de conversations et d'émissions de radio, par exemple) sont, en majorité, des études de cas et, de ce fait, insuffisamment représentatifs pour servir à des recherches comparatives ou typologiques. Boukari (2017, 494, note de bas de page 8) aborde dans sa contribution sur la situation linguistique en Côte d'Ivoire et Burkina Faso le problème récurrent qui apparait au moment de la classification des formes particulières soit comme traits d'une norme endogène ou d'un français mésolectal régional, soit en tant que particularité idiolectale d'un locuteur ou d'une locutrice. Face à cette réalité complexe, surtout là où le français régional n'existe pas comme norme codifiée, la question se pose de savoir si la catégorie des particularités linguistiques est vraiment adéquate pour fournir une description qui corresponde à la réalité. Assurément, les français parlés en Afrique se caractérisent par un dynamisme reflétant les besoins de communication dans un contexte multilingue. Les difficultés commencent toutefois lorsqu'on essaie de classifier un phénomène comme 'particularité' d'une variété déterminée (comme le souligne aussi Reutner, voir citation en haut). Pour arriver à une compréhension plus fine de la situation linguistique en Afrique, il semble dès lors plus approprié d'analyser les diverses expressions du français parlé en Afrique sous l'angle d'une théorie générale du langage et de son acquisition, au lieu de vouloir identifier des nouvelles normes émergentes. Cette approche a déjà été défendue, entre autres, par Boutin $(2010,2)$ qui écrit :

La zone [de l'Afrique de l'Ouest, KP] n'est pas choisie pour rechercher des "particularités africaines", mais au contraire à cause de sa pertinence dans l'élaboration d'une théorie générale. En effet, des stratégies communes aux locuteurs francophones de nombreuses zones géographiques y sont plus visibles $d u$ fait qu'une appropriation du français qui n'hérite que partiellement des contraintes de la longue tradition grammaticale de l'écrit.

Une proposition similaire est faite par Ehrhart (p. 714) qui reprend l'idée d'écologie linguistique pour saisir la complexité linguistique en Polynésie et Mélanésie. Pour saisir cette complexité, elle suggère de partir « d'un continuum français - français régional - tayo - langues mélanésiennes, [pour ne pas marquer, KP] les limites entre des systèmes linguistiques, mais plutôt les éléments qui les relient $»$.

Pour conclure, le Manuel des francophonies comble un vide existant depuis longtemps dans les recherches sur l'espace francophone. Il donne un aperçu assez complet du sujet qui sera d'une grande utilité, tant pour la communauté scientifique (chercheurs et chercheuses, étudiant(e)s en linguistique, mais aussi ceux et celles venant d'autres horizons disciplinaires, comme l'histoire, la sociologie ou les sciences de la communication et des médias,) que pour un lectorat plus large intéressé par l'histoire de la langue française et de sa diffusion à travers le monde. Grâce à sa structure claire et homogène, à laquelle s'ajoutent des références détaillées à la fin de chaque chapitre, le livre est susceptible de servir à la fois comme point de départ et comme ressource précieuse pour la poursuite de recherches plus approfondies. Concernant les illustrations du livre, on aurait parfois souhaité qu'elles apparaissent dans un format plus lisible (p.ex. la figure 3 «Typologie de particularités lexicales », p. 47 ou la figure 5 «Diglossie à position en sandwich », p. 53). Mais ce sont des détails. La grande majorité des chapitres rédigés par 36 experts provenant de douze pays est d'une qualité excellente. Cela dit, un usage plus 
sensible et réfléchi aurait été désirable en ce qui concerne la terminologie adoptée dans quelques contributions. Ainsi, l'emploi du terme Afrique noire, bien qu'il ait été très répandu à partir des années 1970 dans les travaux sur les différentes expressions linguistiques du français parlé en Afrique, semble aujourd'hui tout à fait inapproprié, d'autant plus que l'expression Afrique subsaharienne est une alternative plus neutre et moins connotée. L'équivalent allemand 'Schwarzafrika' fait désormais partie d'un vocabulaire considéré inacceptable même dans le langage quotidien. De ce fait, le recours à une telle notion (par exemple dans l'article « Normes endogènes, variétés de prestige et pluralité normative » (p. 65-86)) apparait pour le moins surprenant. Remarquons, par ailleurs, que l'éditrice de l'ouvrage ne l'utilise jamais dans ses contributions. Cet aspect métalinguistique mérite d'être mentionné dans la mesure où il nous rappelle la persistance manifeste de relations de domination et d'inégalité dans l'espace francophone de nos jours. Une réévaluation critique de nos habitudes linguistiques ne modifiera certainement pas cette réalité du jour au lendemain, mais elle ne constitue pas moins un premier pas vers un changement de notre perception de l'autre dans un monde pluriel.

\section{Références}

Boutin Béatrice Akissi, 2010, «Traces de l'énonciateur dans le discours rapporté : les particules énonciatives et que indicateurs de quelle parole? ", Communication du IV $\mathrm{Ci}$-Dit Colloque international, Nice, 11-13 juin 2009 http://revel.unice.fr/symposia/cidit/index.html?id=384, dernière consultation le 7 octobre 2020.

Robillard Didier de, Beniamino Michel (dir.), 1993, Le français dans l'espace francophone, Tome 1, Paris : Champion.

Robillard Didier de, Beniamino Michel (dir.), 1996, Le français dans l'espace francophone, Tome 2, Paris : Champion.

Valdman Albert (dir.), 1979, Le français hors de France, Paris : Champion. 


\section{GLOTTOPOL}

Revue de sociolinguistique en ligne

Comité de rédaction : Michaël Abecassis (University of Oxford), Salih Akin (Université de Rouen Normandie), Sophie Babault (Université de Lille), Aude Bretegnier (Université du Mans), Claude Caitucoli, Véronique Castellotti (Université de Tours), Régine DelamotteLegrand (Université de Rouen Normandie), Alexandre Duchêne (Université de Fribourg), Valentin Feussi (Université d'Angers), Robert Fournier (Carleton University, Ottawa), Stéphanie Galligani (Université Grenoble Alpes), Médéric Gasquet-Cyrus (Université AixMarseille), Emmanuelle Huver (Université de Tours), Normand Labrie (Université de Toronto), Foued Laroussi (Université de Rouen Normandie), Benoit Leblanc (Université du Québec à Trois-Rivières), Mylène Lebon-Eyquem (Université de la Réunion), Fabienne Leconte (Université de Rouen Normandie), Gudrun Ledegen (Université de Rennes), Danièle Moore (Simon Fraser University, Vancouver), Clara Mortamet (Université Jean Monnet, Saint Etienne), Alioune Ndao (Université Cheik Anta Diop, Dakar), Isabelle Pierozak (Université de Tours), Cécile Van den Avenne (Université Sorbonne Nouvelle, Paris 3).

Rédactrice en chef : Clara Mortamet.

Directrice de publication : Fabienne Leconte.

Comité scientifique: Michelle Auzanneau (Université de Paris), Margaret Bento (Université de Paris), Jacqueline Billiez (Université de Grenobles Alpes), Philippe Blanchet (Université de Rennes), Jean-Michel Eloy (Université d'Amiens), Françoise Gadet (Université Paris Nanterre), Monica Heller (Université de Moncton), Caroline Juillard (Université de Paris), Jean-Marie Klinkenberg (Université de Liège), Marinette Matthey (Université Grenoble Alpes), Marie-Louise Moreau (Université de Mons-Hainault), Robert Nicolaï (Université Côte d'Azur), Didier de Robillard (Université de Tours), Valérie Spaëth (Université Sorbonne nouvelle), Claude Truchot (Université de Strasbourg), Daniel Véronique (Université AixMarseille).

\section{Comité de lecture pour ce numéro :}

Nathalie Auger, Michèle Auzanneau, Margaret Bento, Stéphane Bonnery, Josiane Boutet, Lucile Cadet, Danièle Cogis, Claudine Garcia-Debanc, Marc Debono Régine Delamotte, JeanFrançois De Pietro, Marie-Laure Elalouf, Valentin Feussi, Laurent Gajo, Emmanuelle Huver, Christian Lagarde, Mylène Lebon-Eyquem, Fabienne Leconte, Gudrun Ledegen, Nadia Maillard, Maira Mamede, Bruno Maurer, Clara Mortamet, Fanny Rink, Valerie Spaëth, Marielle Rispail, Françoise Ropé, Eguzki Urteaga, Cécile Van den Avenne, Daniel Véronique 